Research Article

\title{
On Distance-Based Topological Descriptors of Chemical Interconnection Networks
}

\author{
Min Hu, ${ }^{1}$ Haidar Ali $\mathbb{D}^{2}$ Muhammad Ahsan Binyamin $\mathbb{D}^{2},{ }^{2}$ Bilal Ali, ${ }^{2}$ Jia-Bao Liu $\left(\mathbb{D},{ }^{3}\right.$ \\ and Chengmei Fan ${ }^{4}$
}

${ }^{1}$ School of Economics and Management, Anhui Normal University, Wuhu 241002, China
${ }^{2}$ Department of Mathematics, Government College University, Faisalabad, Pakistan
${ }^{3}$ School of Mathematics and Physics, Anhui Jianzhu University, Hefei 230601, China
${ }^{4}$ College of Modern Service Industry, Hefei College of Finance and Economics, Hefei 230601, China

Correspondence should be addressed to Muhammad Ahsan Binyamin; ahsanbanyamin@gmail.com

Received 8 January 2021; Revised 24 February 2021; Accepted 1 March 2021; Published 16 March 2021

Academic Editor: Ghulam Mustafa

Copyright ( $2021 \mathrm{Min} \mathrm{Hu}$ et al. This is an open access article distributed under the Creative Commons Attribution License, which permits unrestricted use, distribution, and reproduction in any medium, provided the original work is properly cited.

\begin{abstract}
Structure-based topological descriptors of chemical networks enable us the prediction of physico-chemical properties and the bioactivities of compounds through QSAR/QSPR methods. Topological indices are the numerical values to represent a graph which characterises the graph. One of the latest distance-based topological index is the Mostar index. In this paper, we study the Mostar index, Szeged index, PI index, $\mathrm{ABC}_{\mathrm{GG}}$ index, and NGG index, for chain oxide network $\mathrm{COX}_{n}$, chain silicate network $\mathrm{CS}_{n}$, ortho chain $S_{n}$, and para chain $Q_{n}$, for the first time. Moreover, analytically closed formulae for these structures are determined.
\end{abstract}

\section{Introduction and Preliminary Results}

All the graphs $G$ in this paper are considered to be finite, undirected, and loopless. Graph $G$ is the set made up of vertices (also called the nodes) which are connected with the edges (also called links). It consists on two sets $V$ and $E$, where $V$ is called the vertex set and $E$ is called the edge set. In order to understand the properties and information contained in the connectivity pattern of graphs, there are many numbers of numerical quantities, known as structure invariants, topological indices, or topological descriptors, which have been derived and studied over the past few decades. The topological indices have vast number of applications in the chemical graph theory which is the special branch of mathematical chemistry.Graph theory has a wide range of applications in engineering due to its diagrammatic nature. It is used in computer science to study the algorithms and flow of information. In engineering, it is used to model the graphics and designs of different networks by converting them in the form of graph.
The topological indices are very much used for characterizing the chemical graphs on the basis of their numerical values. They establish the relationship between the structure and properties of molecule. Topological indices are widely used in QSAR and QSPR research studies [1]. Till now, many topological indices have been derived. For any two graphs $G$ and $H$ which are isomorphic to each other, then $\operatorname{Top}(G)=\operatorname{Top}(H)$ [2]. Due to the success of simple topological indices, such as Wiener Index [3], Zagreb index [4], and Szeged index [5], motivated others, hundreds of topological indices are introduced. Wiener index is one of the first index which was introduced by Harold Wiener in 1947 [6], when he was working on the boiling point of paraffins. The Wiener index [7] of a graph $G$ is defined as the sum of all the distances between pairs of vertices of $G$ :

$$
W(G)=\sum_{(u, v) \subseteq V(G)} d(u, v),
$$

where $d(u, v)$ denotes the shortest-path distance in $G$.

The Szeged index is defined as 


$$
S_{z}(G)=\sum_{e=u v \in E(G)} n_{u} n_{v}
$$

where $n_{u}$ denotes the number of vertices of $G$ closer to $u$ than to $v$ and $n_{v}$ is defined as the number of vertices of $G$ closer to $v$ than to $u$. This was first studied by Gutman. Later, it is known as the Szeged index [8].

The PI index [9], of a graph $G$, is defined as

$$
P I_{v}(G)=\sum_{e=u v \in E(G)} n_{u}+n_{v} \text {. }
$$

The Graovac-Ghorbani index is defined as

$$
\mathrm{ABC}_{\mathrm{GG}}(G)=\sum_{e=u v \in E(G)} \sqrt{\frac{n_{u}+n_{v}-2}{n_{u} n_{v}}},
$$

and this index is introduced by Graovac and Ghorbani [10], and Furtula [11] used the name Graovac-Ghorbani index.

The normalized $\mathrm{ABC}_{\mathrm{GG}}$ index is NGG index, first studied by Dimitrov et al. [12], and is defined as

$$
\operatorname{NGG}(G)=\sum_{e=u v \in E(G)} \frac{1}{\sqrt{n_{u} n_{v}}} .
$$

A chemical graph is a simple graph in which atoms correspond to the vertices and edge denotes the bond between two atoms. A topological index, specially, the Mostar index is one of the latest topological index, derived in 2018 [13]. Previously, Arockiaraj [14] found the Mostar indices of carbon nanostructures, and Hayata and Zhou [15] calculated the large Mostar index on cacti. The Mostar index for a graph $G$ is defined as the sum of all the absolutes values of the difference between $n_{u}$ and $n_{v}$, where $u$ and $v$ are the adjacent vertices of an edge:

$$
\operatorname{Mo}(G)=\sum_{e=u v \in E(G)}\left|n_{u}-n_{v}\right| .
$$

\section{Main Results}

The main goal of this article is to compute the Mostar index of ortho chain and para chain using the edge cut method; also, we find the Mostar index, Szeged index, PI index, $\mathrm{ABC}_{\mathrm{GG}}$ index, and NGG index of oxide chains, chain silicates, ortho chain, and para chain by using the technique of edge partition. The notations used in this paper are standard and taken from the book of west [16]. For the concepts and terms not defined here, we refer the reader to concern with the book of Harary [17] and also concern with [18-25].

2.1. Results for the Chain Oxide Network COX ${ }_{n}$. In this section, we discuss $\mathrm{COX}_{n}$ and compute the exact results for Szeged, PI, $\mathrm{ABC}_{\mathrm{GG}}, \mathrm{NGG}$, and Mostar index. If we remove the silicon atom from the silicate network, then the resulting

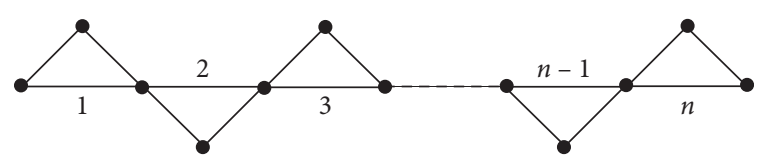

Figure 1: Oxide network.

network is an oxide network [26], which consists of three oxygen atoms. Oxide network has the triangular structure. If an oxide network shares its oxygen with other oxide network linearly, then the oxide chain is formed, as shown in Figure 1.

Theorem 1. Let $G_{1}$ be the oxide network of $n$ order, then its Szeged index is $2 n^{3}+6 n^{2}+n / 3$.

Proof. Let $G_{1} \cong \mathrm{OX}(n)$, where $n \geq 2$; also, $n$ is an integer.

$$
\begin{aligned}
& S z\left(G_{1}\right)=\sum_{e \in E\left(G_{1}\right)} n_{u} n_{v}, \\
& S z\left(G_{1}\right)=\sum_{e \in E\left(G_{1}\right)} n_{2} n_{2}+\sum_{e \in E\left(G_{1}\right)} n_{2} n_{4}+\sum_{e \in E\left(G_{1}\right)} n_{4} n_{4} .
\end{aligned}
$$

By using Table 1, we have

$$
\begin{aligned}
& S z\left(G_{1}\right)=2+2 n^{2}+4 n-4+\frac{2 n^{3}-11 n+6}{3}, \\
& S z\left(G_{1}\right)=\frac{2 n^{3}+6 n^{2}+n}{3},
\end{aligned}
$$

which is required.

Theorem 2. Let $G_{1}$ be the oxide network of $n$ order; then, its $P I$ index is $4 n^{2}+2 n$.

Proof. Let $G_{1} \cong \mathrm{OX}(n)$, where $n \geq 2$; also, $n$ is an integer:

$$
\begin{aligned}
\mathrm{PI}_{v}\left(G_{1}\right) & =\sum_{e \in E\left(G_{1}\right)} n_{u}+n_{v}, \\
\mathrm{PI}_{v}\left(G_{1}\right) & =\sum_{e \in E\left(G_{1}\right)} n_{2}+n_{2}+\sum_{e \in E\left(G_{1}\right)} n_{2}+n_{4}+\sum_{e \in E\left(G_{1}\right)} n_{4}+n_{4} .
\end{aligned}
$$

By using Table 2, we have

$$
\begin{aligned}
& \mathrm{PI}_{v}\left(G_{1}\right)=4+2 n^{2}+6 n-4+2 n^{2}-4 n, \\
& \mathrm{PI}_{v}\left(G_{1}\right)=4 n^{2}+2 n,
\end{aligned}
$$

which is required.

Theorem 3. Let $G_{1}$ be the oxide network of $n$ order; then, its $A B C_{G G}$ index is $1+\sqrt{n^{2}+3 n-3 / n^{2}+2 n-2}+$ $\sqrt{6 n^{2}-12 n-6 / 2 n^{3}-11 n+6}$. 
TABLE 1: Edge partition of oxide network of $n$ order.

\begin{tabular}{lcc}
\hline Edge partition $d_{u}, d_{v}$ & Number of edges & Szeged index \\
\hline 2,2 & 2 & 2 \\
2,4 & $2 n$ & $2 n^{2}+4 n-4$ \\
4,4 & $n-2$ & $2 n^{3}-11 n+6 / 3$ \\
\hline
\end{tabular}

TABLE 2: Edge partition of oxide network of $n$ order.

\begin{tabular}{lcc}
\hline Edge partition $d_{u}, d_{v}$ & Number of edges & PI index \\
\hline 2,2 & 2 & 4 \\
2,4 & $2 n$ & $2 n^{2}+6 n-4$ \\
4,4 & $n-2$ & $2 n^{2}-4 n$ \\
\hline
\end{tabular}

Proof. Let $G_{1} \cong \mathrm{OX}(n)$, where $n \geq 2$; also, $n$ is an integer:
TABLE 3: Edge partition of oxide network of $n$ order.

\begin{tabular}{lcc}
\hline $\begin{array}{l}\text { Edge partition } \\
d_{u}, d_{v}\end{array}$ & Number of edges & $\mathrm{ABC}_{\mathrm{GG}}$ index \\
\hline 2,2 & 2 & 1 \\
2,4 & $2 n$ & $\sqrt{n^{2}+3 n-3 / n^{2}+2 n-2}$ \\
4,4 & $n-2$ & $\sqrt{6 n^{2}-12 n-6 / 2 n^{3}-11 n+6}$ \\
\hline
\end{tabular}

TABLE 4: Edge partition of oxide network of $n$ order.

\begin{tabular}{lcc}
\hline Edge partition $d_{u}, d_{v}$ & Number of edges & NGG index \\
\hline 2,2 & 2 & $1 / \sqrt{2}$ \\
2,4 & $2 n$ & $1 / \sqrt{2 n^{2}+4 n-4}$ \\
4,4 & $n-2$ & $\sqrt{3 / 2 n^{3}-11 n+6}$ \\
\hline
\end{tabular}

$$
\begin{aligned}
\operatorname{ABC}_{\mathrm{GG}}\left(G_{1}\right) & =\sum_{e \in E\left(G_{1}\right)} \sqrt{\frac{n_{u}+n_{v}-2}{n_{u} n_{v}}}, \\
\mathrm{ABC}_{\mathrm{GG}}\left(G_{1}\right) & =\sum_{e \in E\left(G_{1}\right)} \sqrt{\frac{n_{2}+n_{2}-2}{n_{2} n_{2}}}+\sum_{e \in E\left(G_{1}\right)} \sqrt{\frac{n_{2}+n_{4}-2}{n_{2} n_{4}}}+\sum_{e \in E\left(G_{1}\right)} \sqrt{\frac{n_{4}+n_{4}-2}{n_{4} n_{4}}} .
\end{aligned}
$$

By using Table 3, we have

$$
\mathrm{ABC}_{\mathrm{GG}}\left(G_{1}\right)=1+\sqrt{\frac{n^{2}+3 n-3}{n^{2}+2 n-2}}+\sqrt{\frac{6 n^{2}-12 n-6}{2 n^{3}-11 n+6}},
$$

which is required.

Theorem 4. Let $G_{1}$ be the oxide network of $n$ order; then, its $N G G\left(G_{1}\right) \quad$ index is $1 / \sqrt{2}+1 / \sqrt{2 n^{2}+4 n-4}+$ $\sqrt{3 / 2 n^{3}-11 n+6}$.

Proof. Let $G_{1} \cong \mathrm{OX}(n)$, where $n \geq 2$; also, $n$ is an integer:

$$
\begin{aligned}
& \operatorname{NGG}\left(G_{1}\right)=\sum_{e \in E\left(G_{1}\right)} \frac{1}{\sqrt{n_{u} n_{v}}}, \\
& \operatorname{NGG}\left(G_{1}\right)=\sum_{e \in E\left(G_{1}\right)} \frac{1}{\sqrt{n_{2} n_{2}}}+\sum_{e \in E\left(G_{1}\right)} \frac{1}{\sqrt{n_{2} n_{4}}}+\sum_{e \in E\left(G_{1}\right)} \frac{1}{\sqrt{n_{4} n_{4}}} .
\end{aligned}
$$

By using Table 4, we have

$$
\operatorname{NGG}\left(G_{1}\right)=\frac{1}{\sqrt{2}}+\frac{1}{\sqrt{2 n^{2}+4 n-4}}+\sqrt{\frac{3}{2 n^{3}-11 n+6}}
$$

which is required.

Theorem 5. Let $G_{1}$ be the oxide network of even order; then, its Mostar index is $3 n^{2}-2 n$.

Proof. Let $G_{1} \cong \mathrm{OX}(n)$, where $n \geq 2$; also, $n$ is even:

$$
\begin{aligned}
& \operatorname{Mo}\left(G_{1}\right)=\sum_{u v \in E\left(G_{1}\right)}\left|n_{u}-n_{v}\right|, \\
& \operatorname{Mo}\left(G_{1}\right)=\sum_{u v \in E\left(G_{1}\right)}\left|n_{2}-n_{2}\right|+\sum_{u v \in E\left(G_{1}\right)}\left|n_{2}-n_{4}\right|+\sum_{u v \in E\left(G_{1}\right)}\left|n_{4}-n_{4}\right| .
\end{aligned}
$$

By using Table 5, we have

$$
\begin{aligned}
& \operatorname{Mo}\left(G_{1}\right)=0+2 n^{2}+2 n-4+n^{2}-4 n+4, \\
& \operatorname{Mo}\left(G_{1}\right)=3 n^{2}-2 n,
\end{aligned}
$$

which is required.

Theorem 6. Let $G_{1}$ be the oxide network of odd order; then, its Mostar index is $3 n^{2}-2 n-1$.

Proof. Let $G_{1} \cong \mathrm{OX}(n)$, where $n \geq 1$; also, $n$ is odd:

$$
\begin{aligned}
& \operatorname{Mo}\left(G_{1}\right)=\sum_{u v \in E\left(G_{1}\right)} n_{u}-n_{v}, \\
& \operatorname{Mo}\left(G_{1}\right)=\sum_{u v \in E\left(G_{1}\right)}\left|n_{2}-n_{2}\right|+\sum_{u v \in E\left(G_{1}\right)}\left|n_{2}-n_{4}\right|+\sum_{u v \in E\left(G_{1}\right)}\left|n_{4}-n_{4}\right| .
\end{aligned}
$$

By using Table 6, we have

$$
\begin{aligned}
& \operatorname{Mo}\left(G_{1}\right)=0+2 n^{2}+2 n-4+n^{2}-4 n+3, \\
& \operatorname{Mo}\left(G_{1}\right)=3 n^{2}-2 n-1,
\end{aligned}
$$

which is required.

2.2. Results for the Chain Silicate Network $C S_{n}$. In this section, we discuss $\mathrm{CS}_{n}$ and compute the exact results for Szeged, PI, $\mathrm{ABC}_{\mathrm{GG}}, \mathrm{NGG}$, and Mostar index. Silicates are the 
TABLE 5: Edge partition of oxide network of even order.

\begin{tabular}{lcc}
\hline Edge partition $d_{u}, d_{v}$ & Number of edges & Mostar index \\
\hline 2,2 & 2 & 0 \\
2,4 & $2 n$ & $2 n^{2}+2 n-4$ \\
4,4 & $n-2$ & $n^{2}-4 n+4$ \\
\hline
\end{tabular}

TABLE 6: Edge partition of oxide network of odd order.

\begin{tabular}{lcc}
\hline Edge partition $d_{u}, d_{v}$ & Number of edges & Mostar index \\
\hline 2,2 & 2 & 0 \\
2,4 & $2 n$ & $2 n^{2}+2 n-4$ \\
4,4 & $n-2$ & $n^{2}-4 n+3$ \\
\hline
\end{tabular}

compounds which consist of silicon and oxygen, having the tetrahedron structure with bond angle of $109.5^{\circ} . \mathrm{SiO}_{4}$ is found in almost all of the silicates. A single tetrahedron has a shape like a pyramid with triangular base. It has four oxygen atoms at its corners, and silicon atom is bounded equally with oxygen atoms with bond length of $162 \mathrm{pm}$. A single tetrahedron is shown in Figure 2(a). If a single tetrahedron shares its oxygen with other tetrahedrons; then, a linear silicate chain [27] is formed, as shown in Figure 2(b).

Theorem 7. Let $G_{2}$ be the chain silicate network of $n$ order; then, its Szeged index is $3 n^{3}+9 n^{2} / 2$.

Proof. Let $G_{2} \cong C S_{n}$, where $n \geq 2$; also, $n$ is an integer:

$$
\begin{aligned}
\operatorname{Sz}\left(G_{2}\right) & =\sum_{e \in E\left(G_{2}\right)} n_{u} n_{v}, \\
\mathrm{Sz}\left(G_{2}\right) & =\sum_{e \in E\left(G_{2}\right)} n_{3} n_{3}+\sum_{e \in E\left(G_{2}\right)} n_{3} n_{6}+\sum_{e \in E\left(G_{2}\right)} n_{6} n_{6} .
\end{aligned}
$$

$$
\begin{aligned}
\operatorname{ABC}_{\mathrm{GG}}\left(G_{2}\right) & =\sum_{e \in E\left(G_{2}\right)} \sqrt{\frac{n_{u}+n_{v}-2}{n_{u} n_{v}}}, \\
\mathrm{ABC}_{\mathrm{GG}}\left(G_{2}\right) & =\sum_{e \in E\left(G_{2}\right)} \sqrt{\frac{n_{3}+n_{3}-2}{n_{3} n_{3}}}+\sum_{e \in E\left(G_{2}\right)} \sqrt{\frac{n_{3}+n_{6}-2}{n_{3} n_{6}}}+\sum_{e \in E\left(G_{2}\right)} \sqrt{\frac{n_{6}+n_{6}-2}{n_{6} n_{6}}} .
\end{aligned}
$$

By using Table 7, we have

$$
\begin{aligned}
& \operatorname{Sz}\left(G_{2}\right)=n+4+6 n^{2}+4 n-8+\frac{3 n^{3}-3 n^{2}-10 n+8}{2}, \\
& \operatorname{Sz}\left(G_{2}\right)=\frac{3 n^{3}+9 n^{2}}{2},
\end{aligned}
$$

which is required.

Theorem 8. Let $G_{2}$ be the chain silicate network of $n$ order; then, its $P I$ index is $9 n^{2}+3 n$.

Proof. Let $G_{2} \cong \mathrm{CS}_{n}$, where $n \geq 2$; also, $n$ is an integer:

$$
\begin{aligned}
& \operatorname{PI}_{\mathrm{v}}\left(G_{2}\right)=\sum_{e \in E\left(G_{2}\right)} n_{u}+n_{v}, \\
& \operatorname{PI}_{\mathrm{v}}\left(G_{2}\right)=\sum_{e \in E\left(G_{2}\right)} n_{3}+n_{3}+\sum_{e \in E\left(G_{2}\right)} n_{3}+n_{6}+\sum_{e \in E\left(G_{2}\right)} n_{6}+n_{6} .
\end{aligned}
$$

By using Table 8 , we have

$$
\begin{aligned}
& \mathrm{PI}_{\mathrm{v}}\left(G_{2}\right)=2 n+8+6 n^{2}+8 n-10+3 n^{2}-7 n+2, \\
& \mathrm{PI}_{\mathrm{v}}\left(G_{2}\right)=9 n^{2}+3 n,
\end{aligned}
$$

which is required.

Theorem 9. Let $G_{2}$ be the chain silicate network of $n$ order then its $A B C_{G G}$ index is $\sqrt{2 n+6 / n+4}+$ $\sqrt{3 n^{2}+4 n-6 / 3 n^{2}+2 n-4}+\sqrt{6 n^{2}-14 n / 3 n^{3}-3 n^{2}-10 n+8}$.

Proof. Let $G_{2} \cong \mathrm{CS}_{n}$, where $n \geq 2$; also, $n$ is an integer:
By using Table 9, we have

$$
\mathrm{ABC}_{\mathrm{GG}}\left(G_{2}\right)=\sqrt{\frac{2 n+6}{n+4}}+\sqrt{\frac{3 n^{2}+4 n-6}{3 n^{2}+2 n-4}}+\sqrt{\frac{6 n^{2}-14 n}{3 n^{3}-3 n^{2}-10 n+8}},
$$

which is required.

Theorem 10. Let $G_{2}$ be the chain silicate network of $n$ order; then, its NGG index is $1 / \sqrt{n+4}+1 / \sqrt{6 n^{2}+4 n-8}$ $+\sqrt{2 / 3 n^{3}-3 n^{2}-10 n+8}$.

Proof. Let $G_{2} \cong \mathrm{CS}_{n}$, where $n \geq 2$; also, $n$ is an integer: 


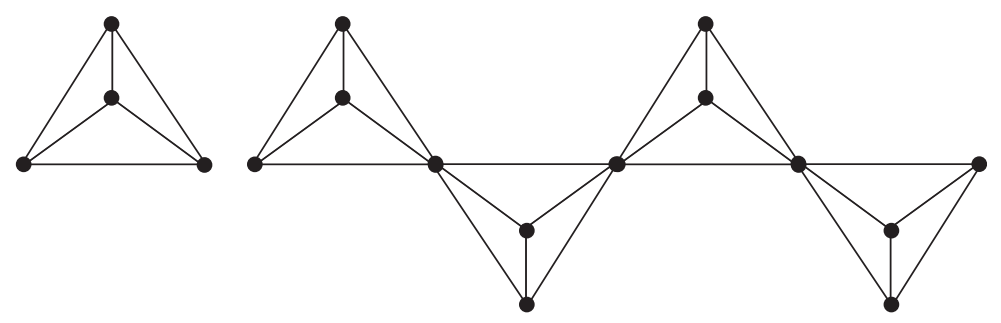

(a)

(b)

Figure 2: (a) Single silicate and (b) chain silicate.

TABLE 7: Edge partition of chain silicate network of $n$ order.

\begin{tabular}{lcc}
\hline Edge partition $d_{u}, d_{v}$ & Number of edges & Szeged index \\
\hline 3,3 & $n+4$ & $n+4$ \\
3,6 & $2(2 n-1)$ & $6 n^{2}+4 n-8$ \\
6,6 & $n-2$ & $3 n^{3}-3 n^{2}-10 n+8 / 2$ \\
\hline
\end{tabular}

TABLE 8: Edge partition of chain silicate network of $n$ order.

\begin{tabular}{lcc}
\hline Edge partition $d_{u}, d_{v}$ & Number of edges & PI index \\
\hline 3,3 & $n+4$ & $2 \mathrm{n}+8$ \\
3,6 & $2(2 n-1)$ & $6 n^{2}+8 n-10$ \\
6,6 & $n-2$ & $3 n^{2}-7 n+2$ \\
\hline
\end{tabular}

TABLE 9: Edge partition of chain silicate network of $n$ order.

\begin{tabular}{lcc}
\hline Edge partition $d_{u}, d_{v}$ & Number of edges & $\mathrm{ABC}_{\mathrm{GG}}$ index \\
\hline 3,3 & $n+4$ & $\sqrt{2 n+6 / n+4}$ \\
3,6 & $2(2 n-1)$ & $\sqrt{3 n^{2}+4 n-6 / 3 n^{2}+2 n-4}$ \\
6,6 & $n-2$ & $\sqrt{6 n^{2}-14 n / 3 n^{3}-3 n^{2}-10 n+8}$ \\
\hline
\end{tabular}

TABLE 10: Edge partition of chain silicate network of $n$ order.

\begin{tabular}{lcc}
\hline Edge partition $d_{u}, d_{v}$ & Number of edges & $\mathrm{ABC}_{\mathrm{GG}}$ index \\
\hline 3,3 & $n+4$ & $1 / \sqrt{n+4}$ \\
3,6 & $2(2 n-1)$ & $1 / \sqrt{6 n^{2}+4 n-8}$ \\
6,6 & $n-2$ & $\sqrt{2 / 3 n^{3}-3 n^{2}-10 n+8}$ \\
\hline
\end{tabular}

which is required.

Theorem 11. Let $G_{2}$ be the chain silicate network of even order; then, its Mostar index is $15 n^{2}-12 n / 2$.

Proof. Let $G_{2} \cong \mathrm{CS}_{n}$, where $n \geq 2$; also, $n$ is even.

$$
\begin{aligned}
& \operatorname{Mo}\left(G_{2}\right)=\sum_{u v \in E\left(G_{2}\right)}\left|n_{u}-n_{v}\right|, \\
& \operatorname{Mo}\left(G_{2}\right)=\sum_{u v \in E\left(G_{2}\right)}\left|n_{3}-n_{3}\right|+\sum_{u v \in E\left(G_{2}\right)}\left|n_{3}-n_{6}\right|+\sum_{u v \in E\left(G_{2}\right)}\left|n_{6}-n_{6}\right| .
\end{aligned}
$$

By using Table 11, we have

$$
\begin{aligned}
& \operatorname{Mo}\left(G_{2}\right)=0+6 n^{2}-6+\frac{3 n^{2}-12 n+12}{2}, \\
& \operatorname{Mo}\left(G_{2}\right)=\frac{15 n^{2}-12 n}{2}
\end{aligned}
$$

TABLE 11: Edge partition of chain silicate network of even order.

\begin{tabular}{lcc}
\hline Edge partition $d_{u}, d_{v}$ & Number of edges & Mostar index \\
\hline 3,3 & $n+4$ & 0 \\
3,6 & $2(2 n-1)$ & $6 n^{2}-6$ \\
6,6 & $n-2$ & $3 n^{2}-12 n+12 / 2$ \\
\hline
\end{tabular}

which is required.

Theorem 12. Let $G_{2}$ be the chain silicate network of odd order; then, its Mostar index is $15 n^{2}-12 n-3 / 2$.

Proof. Let $G_{2} \cong \mathrm{CS}_{n}$, where $n \geq 1$; also, $n$ is odd:

$$
\begin{aligned}
& \operatorname{Mo}\left(G_{2}\right)=\sum_{u v \in E\left(G_{2}\right)}\left|n_{u}-n_{v}\right|, \\
& \operatorname{Mo}\left(G_{2}\right)=\sum_{u v \in E\left(G_{2}\right)}\left|n_{3}-n_{3}\right|+\sum_{u v \in E\left(G_{2}\right)}\left|n_{3}-n_{6}\right|+\sum_{u v \in E\left(G_{2}\right)}\left|n_{6}-n_{6}\right| .
\end{aligned}
$$

By using Table 12, we have

$$
\begin{aligned}
& \operatorname{Mo}\left(G_{2}\right)=0+6 n^{2}-6+\frac{3 n^{2}-12 n+9}{2}, \\
& \operatorname{Mo}\left(G_{2}\right)=\frac{15 n^{2}-12 n-3}{2},
\end{aligned}
$$


TABLE 12: Edge partition of chain silicate network of odd order.

\begin{tabular}{lcc}
\hline Edge partition $d_{u}, d_{v}$ & Number of edges & Mostar index \\
\hline 3,3 & $n+4$ & 0 \\
3,6 & $2(2 n-1)$ & $6 n^{2}-6$ \\
6,6 & $n-2$ & $3 n^{2}-12 n+9 / 2$ \\
\hline
\end{tabular}

which is required.

2.3. Results for the Ortho Chain $S_{n}$. In this section, we discuss $S_{n}$ and compute the exact results for Szeged, $\mathrm{PI}, \mathrm{ABC}_{\mathrm{GG}}$, NGG, and Mostar index. The single molecule of para and ortho chain has the same structure. Basically, it is a cycle graph having 4 sides denoted as $C_{4}$ and represented as a four-sided regular polygon. The ortho chain has a zig-zag structure where each corner of $C_{4}$ is attached linearly, as shown in Figure 3. The para chain has a structure in which each $C_{4}$ is attached at corner to corner with other $C_{4}$ but not linearly, as shown in Figure 4 [28].

Theorem 13. Let $G_{3}$ be the ortho chain of $n$ order; then, its Szeged index is $3 n^{3}+15 n^{2}-2 n$.

Proof. Let $G_{3} \cong S_{n}$, where $n \geq 2$; also, $n$ is an integer.

$$
\begin{aligned}
& \operatorname{Sz}\left(G_{3}\right)=\sum_{e \in E\left(G_{3}\right)} n_{u} n_{v}, \\
& \operatorname{Sz}\left(G_{3}\right)=\sum_{e \in E\left(G_{3}\right)} n_{2} n_{2}+\sum_{e \in E\left(G_{3}\right)} n_{2} n_{4}+\sum_{e \in E\left(G_{3}\right)} n_{4} n_{4} .
\end{aligned}
$$

By using Table 13, we have

$$
\begin{aligned}
& \operatorname{Sz}\left(G_{3}\right)=\frac{3 n^{3}+3 n^{2}+26 n-8}{2}+12 n^{2}-4 n+\frac{3 n^{3}+3 n^{2}-22 n+8}{2}, \\
& \operatorname{Sz}\left(G_{3}\right)=3 n^{3}+15 n^{2}-2 n,
\end{aligned}
$$

which is required.

Theorem 14. Let $G_{3}$ be the ortho chain of norder; then, its PI index is $12 n^{2}+4 n$.

Proof. Let $G_{3} \cong S_{n}$, where $n \geq 2$; also, $n$ is an integer:

$$
\begin{aligned}
& \mathrm{PI}_{v}\left(G_{3}\right)=\sum_{e \in E\left(G_{3}\right)} n_{u}+n_{v}, \\
& \mathrm{PI}_{v}\left(G_{3}\right)=\sum_{e \in E\left(G_{3}\right)} n_{2}+n_{2}+\sum_{e \in E\left(G_{3}\right)} n_{2}+n_{4}+\sum_{e \in E\left(G_{3}\right)} n_{4}+n_{4} .
\end{aligned}
$$

By using Table 14, we have

$$
\begin{aligned}
& \mathrm{PI}_{v}\left(G_{3}\right)=3 n^{2}+7 n+2+6 n^{2}+2 n+3 n^{2}-5 n-2, \\
& \mathrm{PI}_{v}\left(G_{3}\right)=12 n^{2}+4 n,
\end{aligned}
$$

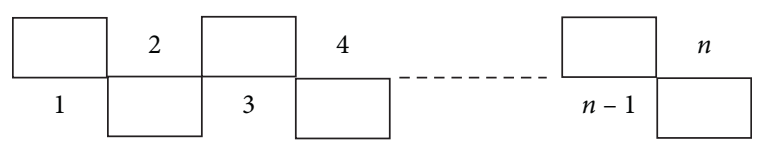

FIGURE 3: Ortho chain of $n$ vertices.

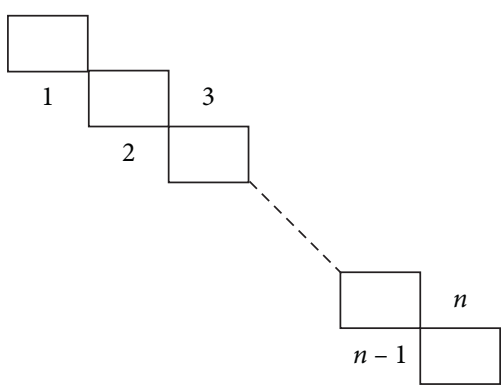

Figure 4: Para chain of $n$ order.

TABLE 13: Edge partition of ortho chain of $n$ order.

\begin{tabular}{lcc}
\hline Edge partition $d_{u}, d_{v}$ & Number of edges & Szeged index \\
\hline 2,2 & $n+2$ & $3 n^{3}+3 n^{2}+26 n-8 / 2$ \\
2,4 & $2 n$ & $12 n^{2}-4 n$ \\
4,4 & $n-2$ & $3 n^{3}+3 n^{2}-22 n+8 / 2$ \\
\hline
\end{tabular}

TABLE 14: Edge partition of ortho chain of $n$ order.

\begin{tabular}{lcc}
\hline Edge partition $d_{u}, d_{v}$ & Number of edges & PI index \\
\hline 2,2 & $n+2$ & $3 n^{2}+7 n+2$ \\
2,4 & $2 n$ & $6 n^{2}+2 n$ \\
4,4 & $n-2$ & $3 n^{2}-5 n-2$ \\
\hline
\end{tabular}

which is required.

Theorem 15. Let $G_{3}$ be the ortho chain of $n$ order; then, its $A B C_{G G} \quad$ index is $\sqrt{6 n^{2}+14 n / 3 n^{3}+3 n^{2}+26 n-8}$ $+\sqrt{3 n^{2}+n-1 / 6 n^{2}-2 n}+\sqrt{6 n^{2}-10 n-8 / 3 n^{3}+3 n^{2}-22 n}$ +8 .

Proof. Let $G_{3} \cong S_{n}$, where $n \geq 2$; also, $n$ is an integer:

$$
\begin{aligned}
\operatorname{ABC}_{\mathrm{GG}}\left(G_{3}\right)= & \sum_{e \in E\left(G_{3}\right)} \sqrt{\frac{n_{u}+n_{v}-2}{n_{u} n_{v}}}, \\
\operatorname{ABC}_{\mathrm{GG}}\left(G_{3}\right)= & \sum_{e \in E\left(G_{3}\right)} \sqrt{\frac{n_{2}+n_{2}-2}{n_{2} n_{2}}}+\sum_{e \in E\left(G_{3}\right)} \sqrt{\frac{n_{2}+n_{4}-2}{n_{2} n_{4}}} \\
& +\sum_{e \in E\left(G_{3}\right)} \sqrt{\frac{n_{4}+n_{4}-2}{n_{4} n_{4}}} .
\end{aligned}
$$

By using Table 15, we have 
TABLE 15: Edge partition of ortho chain of $n$ order.

\begin{tabular}{lcc}
\hline $\begin{array}{l}\text { Edge } \\
\text { partition }\end{array}$ & Number of edges & $A B C_{G G}$ index \\
$d_{u}, d_{v}$ & & \\
\hline 2,2 & $n+2$ & $\sqrt{6 n^{2}+14 n / 3 n^{3}+3 n^{2}+26 n-8}$ \\
2,4 & $2 n$ & $\sqrt{3 n^{2}+n-1 / 6 n^{2}-2 n}$ \\
4,4 & $n-2$ & $\sqrt{6 n^{2}-10 n-8 / 3 n^{3}+3 n^{2}-22 n+8}$ \\
\hline
\end{tabular}

$$
\begin{aligned}
\mathrm{ABC}_{\mathrm{GG}}\left(G_{3}\right)= & \sqrt{\frac{6 n^{2}+14 n}{3 n^{3}+3 n^{2}+26 n-8}}+\sqrt{\frac{3 n^{2}+n-1}{6 n^{2}-2 n}} \\
& +\sqrt{\frac{6 n^{2}-10 n-8}{3 n^{3}+3 n^{2}-22 n+8}}
\end{aligned}
$$

which is required.

Theorem 16. Let $G_{3}$ be the ortho chain of $n$ order; then, its $N G G\left(G_{3}\right)$ index is $\sqrt{2 / 3 n^{3}+3 n^{2}+26 n-8}+1 / 2$ $\sqrt{3 n^{2}-n}+\sqrt{2 / 3 n^{3}+3 n^{2}-22 n+8}$.

Proof. Let $G_{3} \cong S_{n}$, where $n \geq 2$; also, $n$ is an integer:

$$
\begin{aligned}
& \operatorname{NGG}\left(G_{3}\right)=\sum_{e \in E\left(G_{3}\right)} \frac{1}{\sqrt{n_{u} n_{v}}}, \\
& \operatorname{NGG}\left(G_{3}\right)=\sum_{e \in E\left(G_{3}\right)} \frac{1}{\sqrt{n_{2} n_{2}}}+\sum_{e \in E\left(G_{3}\right)} \frac{1}{\sqrt{n_{2} n_{4}}}+\sum_{e \in E\left(G_{3}\right)} \frac{1}{\sqrt{n_{4} n_{4}}} .
\end{aligned}
$$

By using Table 16, we have

$$
\begin{aligned}
\operatorname{NGG}\left(G_{3}\right)= & \sqrt{\frac{2}{3 n^{3}+3 n^{2}+26 n-8}}+\frac{1}{2 \sqrt{3 n^{2}-n}} \\
& +\sqrt{\frac{2}{3 n^{3}+3 n^{2}-22 n+8}}
\end{aligned}
$$

which is required.

Theorem 17. Let $G_{3}$ be the ortho chain of even order; then, its Mostar index is $9 n^{2}-6 n$.

Proof. Let $G_{3} \cong S_{n}$, where $n \geq 2$; also, $n$ is even:

$$
\begin{aligned}
\operatorname{Mo}\left(G_{3}\right)= & \sum_{u v \in E\left(G_{3}\right)}\left|n_{u}-n_{v}\right|, \\
\operatorname{Mo}\left(G_{3}\right)= & \sum_{u v \in E\left(G_{3}\right)}\left|n_{2}-n_{2}\right|+\sum_{u v \in E\left(G_{3}\right)}\left|n_{2}-n_{4}\right| \\
& +\sum_{u v \in E\left(G_{3}\right)}\left|n_{4}-n_{4}\right| .
\end{aligned}
$$

By using Table 17, we have
TABLE 16: Edge partition of oxide network of $n$ order.

\begin{tabular}{lcc}
\hline Edge partition $d_{u}, d_{v}$ & Number of edges & NGG index \\
\hline 2,2 & $n+2$ & $\sqrt{2 / 3 n^{3}+3 n^{2}+26 n-8}$ \\
2,4 & $2 n$ & $1 / 2 \sqrt{3 n^{2}-n}$ \\
4,4 & $n-2$ & $\sqrt{2 / 3 n^{3}+3 n^{2}-22 n+8}$ \\
\hline
\end{tabular}

TABLE 17: Edge partition of ortho chain of even order.

\begin{tabular}{lcc}
\hline Edge partition $d_{u}, d_{v}$ & Number of edges & Mostar index \\
\hline 2,2 & $n+2$ & $3 n^{2}+12 n-12 / 2$ \\
2,4 & $2 n$ & $6 n^{2}-6 n$ \\
4,4 & $n-2$ & $3 n^{2}-12 n+12 / 2$ \\
\hline
\end{tabular}

$$
\begin{aligned}
& \operatorname{Mo}\left(G_{3}\right)=\frac{3 n^{2}+12 n-12}{2}+6 n^{2}-6 n+\frac{3 n^{2}-12 n+12}{2}, \\
& \operatorname{Mo}\left(G_{3}\right)=9 n^{2}-6 n,
\end{aligned}
$$

which is required.

Theorem 18. Let $G_{3}$ be the ortho chain of odd order; then, its Mostar index is $9 n^{2}-6 n-3$.

Proof. Let $G_{3} \cong S_{n}$, where $n \geq 1$; also, $n$ is odd:

$$
\begin{aligned}
\operatorname{Mo}\left(G_{3}\right)= & \sum_{u v \in E\left(G_{3}\right)}\left|n_{u}-n_{v}\right|, \\
\operatorname{Mo}\left(G_{3}\right)= & \sum_{u v \in E\left(G_{3}\right)}\left|n_{2}-n_{2}\right|+\sum_{u v \in E\left(G_{3}\right)}\left|n_{2}-n_{4}\right| \\
& +\sum_{u v \in E\left(G_{3}\right)}\left|n_{4}-n_{4}\right| .
\end{aligned}
$$

By using Table 18, we have

$$
\begin{aligned}
& \operatorname{Mo}\left(G_{3}\right)=\frac{3 n^{2}+12 n-15}{2}+6 n^{2}-6 n+\frac{3 n^{2}-12 n+9}{2}, \\
& \operatorname{Mo}\left(G_{3}\right)=9 n^{2}-6 n-3
\end{aligned}
$$

which is required.

2.4. Results for the Para Chain $Q_{n}$. In this section, we discuss $Q_{n}$ and compute the exact results for Szeged, PI, ABC $\mathrm{GG}_{\mathrm{G}}$, NGG, and Mostar index.

Theorem 19. Let $G_{4}$ be the para chain of $n$ order; then, its Szeged index is $6 n^{3}+6 n^{2}+4 n$.

Proof. Let $G_{4} \cong Q_{n}$, where $n \geq 2$; also, $n$ is an integer. 
TABLE 18: Edge partition of ortho chain of odd order.

\begin{tabular}{lcc}
\hline Edge partition $d_{u}, d_{v}$ & Number of edges & Mostar index \\
\hline 2,2 & $n+2$ & $3 n^{2}+12 n-15 / 2$ \\
2,4 & $2 n$ & $6 n^{2}-6 n$ \\
4,4 & $n-2$ & $3 n^{2}-12 n+9 / 2$ \\
\hline
\end{tabular}

$$
\begin{aligned}
& \operatorname{Sz}\left(G_{4}\right)=\sum_{e \in E\left(G_{4}\right)} n_{u} n_{v}, \\
& \operatorname{Sz}\left(G_{4}\right)=\sum_{e \in E\left(G_{4}\right)} n_{2} n_{2}+\sum_{e \in E\left(G_{4}\right)} n_{2} n_{4} .
\end{aligned}
$$

By using Table 19, we have

$$
\begin{aligned}
& \operatorname{Sz}\left(G_{4}\right)=24 n-8+6 n^{3}+6 n^{2}-20 n+8, \\
& \operatorname{Sz}\left(G_{4}\right)=6 n^{3}+6 n^{2}+4 n,
\end{aligned}
$$

which is required.

Theorem 20. Let $G_{4}$ be the para chain of n order; then, its PI index is $12 n^{2}+4 n$.

Proof. Let $G_{4} \cong Q_{n}$, where $n \geq 2$; also, $n$ is an integer:

$$
\begin{aligned}
& \mathrm{PI}_{v}\left(G_{4}\right)=\sum_{e \in E\left(G_{4}\right)} n_{u}+n_{v}, \\
& \mathrm{PI}_{v}\left(G_{4}\right)=\sum_{e \in E\left(G_{4}\right)} n_{2}+n_{2}+\sum_{e \in E\left(G_{4}\right)} n_{2}+n_{4} .
\end{aligned}
$$

By using Table 20, we have

$$
\begin{aligned}
& \mathrm{PI}_{v}\left(G_{4}\right)=12 n+4+12 n^{2}-8 n-4, \\
& \mathrm{PI}_{v}\left(G_{4}\right)=12 n^{2}+4 n,
\end{aligned}
$$

which is required.

Theorem 21. Let $G_{4}$ be the para chain of $n$ order; then, its $A B C_{G G}$ index $\quad$ is
$+\sqrt{6 n^{2}-4 n-3 / 3 n^{3}+3 n^{2}-10 n+4}$

Proof. Let $G_{4} \cong \mathrm{OX}(n)$, where $n \geq 2$; also, $n$ is an integer:

$$
\begin{aligned}
\operatorname{ABC}_{\mathrm{GG}}\left(G_{4}\right) & =\sum_{e \in E\left(G_{4}\right)} \sqrt{\frac{n_{u}+n_{v}-2}{n_{u} n_{v}}}, \\
\mathrm{ABC}_{\mathrm{GG}}\left(G_{4}\right) & =\sum_{e \in E\left(G_{4}\right)} \sqrt{\frac{n_{2}+n_{2}-2}{n_{2} n_{2}}}+\sum_{e \in E\left(G_{4}\right)} \sqrt{\frac{n_{2}+n_{4}-2}{n_{2} n_{4}}} .
\end{aligned}
$$

By using Table 21, we have

$$
\mathrm{ABC}_{\mathrm{GG}}\left(G_{4}\right)=\sqrt{\frac{6 n+1}{12 n-4}}+\sqrt{\frac{6 n^{2}-4 n-3}{3 n^{3}+3 n^{2}-10 n+4}},
$$

which is required.
TABLE 19: Edge partition of para chain of $n$ order.

\begin{tabular}{lcc}
\hline Edge partition $d_{u}, d_{v}$ & Number of edges & Szeged index \\
\hline 2,2 & 4 & $24 n-8$ \\
2,4 & $4 n-4$ & $6 n^{3}+6 n^{2}-20 n+8$ \\
\hline
\end{tabular}

TABLE 20: Edge partition of para chain of $n$ order.

\begin{tabular}{lcc}
\hline Edge partition $d_{u}, d_{v}$ & Number of edges & PI index \\
\hline 2,2 & 4 & $12 n+4$ \\
2,4 & $4 n-4$ & $12 n^{2}-8 n-4$ \\
\hline
\end{tabular}

TABLE 21: Edge partition of para chain of $n$ order.

\begin{tabular}{lcc}
\hline $\begin{array}{l}\text { Edge } \\
\text { partition } \\
d_{u}, d_{v}\end{array}$ & Number of edges & $\mathrm{ABC}_{\mathrm{GG}}$ index \\
\hline 2,2 & 4 & $\sqrt{6 n+1 / 12 n-4}$ \\
2,4 & $4 n-4$ & $\sqrt{6 n^{2}-4 n-3 / 3 n^{3}+3 n^{2}-10 n+4}$ \\
\hline
\end{tabular}

Theorem 22. Let $G_{4}$ be the para chain of $n$ order; then, its $N G G\left(G_{4}\right)$ index is $1 / 2 \sqrt{6 n-2}+1 / \sqrt{6 n^{3}+6 n^{2}-20 n+8}$.

Proof. Let $G_{4} \cong Q_{n}$, where $n \geq 2$ also $n$ is an integer:

$$
\begin{aligned}
& \operatorname{NGG}\left(G_{4}\right)=\sum_{e \in E\left(G_{4}\right)} \frac{1}{\sqrt{n_{u} n_{v}}}, \\
& \operatorname{NGG}\left(G_{4}\right)=\sum_{e \in E\left(G_{4}\right)} \frac{1}{\sqrt{n_{2} n_{2}}}+\sum_{e \in E\left(G_{4}\right)} \frac{1}{\sqrt{n_{2} n_{4}}} .
\end{aligned}
$$

By using Table 22, we have

$$
\operatorname{NGG}\left(G_{4}\right)=\frac{1}{2 \sqrt{6 n-2}}+\frac{1}{\sqrt{6 n^{3}+6 n^{2}-20 n+8}},
$$

which is required.

Theorem 23. Let $G_{4}$ be the para chain of even order; then, its Mostar index is $6 n^{2}$.

Proof. Let $G_{4} \cong Q_{n}$, where $n \geq 2$; also, $n$ is even:

$$
\begin{aligned}
& \operatorname{Mo}\left(G_{4}\right)=\sum_{u v \in E\left(G_{4}\right)}\left|n_{u}-n_{v}\right|, \\
& \operatorname{Mo}\left(G_{4}\right)=\sum_{u v \in E\left(G_{4}\right)}\left|n_{2}-n_{2}\right|+\sum_{u v \in E\left(G_{4}\right)}\left|n_{2}-n_{4}\right| .
\end{aligned}
$$

By using Table 23, we have

$$
\begin{aligned}
& \operatorname{Mo}\left(G_{4}\right)=12 n-12+6 n^{2}-12 n+12, \\
& \operatorname{Mo}\left(G_{4}\right)=6 n^{2}
\end{aligned}
$$

which is required.

Theorem 24. Let $G_{4}$ be the para chain of odd order; then, its Mostar index is $6 n^{2}-6$. 
TABLE 22: Edge partition of para chain of $n$ order.

\begin{tabular}{lcc}
\hline Edge partition $d_{u}, d_{v}$ & Number of edges & NGG index \\
\hline 2,2 & 4 & $1 / 2 \sqrt{6 n-2}$ \\
2,4 & $4 n-4$ & $1 / \sqrt{6 n^{3}+6 n^{2}-20 n+8}$ \\
\hline
\end{tabular}

TABle 23: Edge partition of para chain of even order.

\begin{tabular}{lcc}
\hline Edge partition $d_{u}, d_{v}$ & Number of edges & Mostar index \\
\hline 2,2 & 4 & $12 n-12$ \\
2,4 & $4 n-4$ & $6 n^{2}-12 n+12$ \\
\hline
\end{tabular}

TABLE 24: Edge partition of para chain of odd order.

\begin{tabular}{lcc}
\hline Edge partition $d_{u}, d_{v}$ & Number of edges & Mostar index \\
\hline 2,2 & 4 & $12 n-12$ \\
2,4 & $4 n-4$ & $6 n^{2}+12 n+6$ \\
\hline
\end{tabular}

TABle 25: Comparison table for chain oxide network.

\begin{tabular}{lcccc}
\hline $\mathrm{OX}(n)$ & $\mathrm{Sz}(G)$ & PI & ABC & NGG \\
\hline 1 & 3 & 6 & - & - \\
2 & 14 & 20 & - & - \\
3 & 37 & 42 & - & - \\
4 & 76 & 72 & 2.75 & 1.04 \\
5 & 135 & 110 & 2.71 & 0.95 \\
6 & 218 & 156 & 2.66 & 0.90 \\
7 & 329 & 210 & 2.62 & 0.86 \\
8 & 472 & 272 & 2.59 & 0.84 \\
9 & 651 & 342 & 2.56 & 0.83 \\
10 & 870 & 420 & 2.53 & 0.81 \\
\hline
\end{tabular}

TABle 26: Comparison table for chain silicate.

\begin{tabular}{lcccc}
\hline $\mathrm{CS}_{n}$ & $\mathrm{Sz}(G)$ & PI & ABC & NGG \\
\hline 1 & 6 & 12 & - & - \\
2 & 30 & 42 & - & - \\
3 & 81 & 90 & - & - \\
4 & 168 & 156 & 2.91 & 0.58 \\
5 & 300 & 240 & 2.89 & 0.49 \\
6 & 486 & 342 & 2.88 & 0.45 \\
7 & 735 & 462 & 2.86 & 0.41 \\
8 & 1056 & 600 & 2.83 & 0.38 \\
9 & 1458 & 756 & 2.81 & 0.35 \\
10 & 1950 & 930 & 2.80 & 0.33 \\
\hline
\end{tabular}

Proof. Let $G_{4} \cong Q_{n}$, where $n \geq 1$; also, $n$ is odd:

$$
\begin{aligned}
& \operatorname{Mo}\left(G_{4}\right) \sum_{u v \in E\left(G_{4}\right)}\left|n_{u}-n_{v}\right|, \\
& \operatorname{Mo}\left(G_{4}\right)=\sum_{u v \in E\left(G_{4}\right)}\left|n_{2}-n_{2}\right|+\sum_{u v \in E\left(G_{4}\right)}\left|n_{2}-n_{4}\right| .
\end{aligned}
$$

By using Table 24, we have

$$
\begin{aligned}
& \operatorname{Mo}\left(G_{4}\right)=12 n-12+6 n^{2}+12 n+6 \\
& \operatorname{Mo}\left(G_{4}\right)=6 n^{2}-6,
\end{aligned}
$$

which is required.
TABLE 27: Comparison table for ortho chain.

\begin{tabular}{lcccc}
\hline$S_{n}$ & Sz $(G)$ & PI & ABC & NGG \\
\hline 1 & 16 & 16 & - & - \\
2 & 80 & 56 & - & - \\
3 & 210 & 120 & - & - \\
4 & 424 & 208 & 1.98 & 0.26 \\
5 & 740 & 320 & 1.88 & 0.19 \\
6 & 1176 & 456 & 1.80 & 0.15 \\
7 & 1750 & 616 & 1.74 & 0.12 \\
8 & 2480 & 800 & 1.68 & 0.10 \\
9 & 3384 & 1006 & 1.63 & 0.09 \\
10 & 4480 & 1240 & 1.58 & 0.08 \\
\hline
\end{tabular}

TABLE 28: Comparison table for para chain.

\begin{tabular}{lcccc}
\hline$Q_{n}$ & $\mathrm{Sz}(G)$ & PI & ABC & NGG \\
\hline 1 & 16 & 16 & - & - \\
2 & 80 & 56 & 1.61 & 0.32 \\
3 & 228 & 120 & 1.46 & 0.20 \\
4 & 496 & 208 & 1.37 & 0.16 \\
5 & 920 & 320 & 1.30 & 0.13 \\
6 & 1536 & 456 & 1.26 & 0.11 \\
7 & 2380 & 616 & 1.22 & 0.10 \\
8 & 3488 & 800 & 1.19 & 0.09 \\
9 & 4896 & 1008 & 1.16 & 0.08 \\
10 & 6640 & 1240 & 1.14 & 0.07 \\
\hline
\end{tabular}

For the comparison of Szeged, PI, $\mathrm{ABC}_{\mathrm{GG}}$, and NGG index of $\mathrm{COX}_{n}$, we computed the indices for different values of $n$. By increasing the values of $n$, we can clearly check from Table 25 that the order of Szeged and PI index is increasing while that of $\mathrm{ABC}_{\mathrm{GG}}$ and $N G G$ is decreasing.

For the comparison of Szeged, PI, $\mathrm{ABC}_{\mathrm{GG}}$, and NGG index of $\mathrm{CS}_{n}$, we computed the indices for different values of $n$. By increasing the values of $n$, we can clearly check from Table 26 that the order of Szeged and PI index is increasing while that of $\mathrm{ABC}_{\mathrm{GG}}$ and $\mathrm{NGG}$ is decreasing.

For the comparison of Szeged, PI, $\mathrm{ABC}_{\mathrm{GG}}$, and NGG index of $S_{n}$, we computed the indices for different values of $n$. By increasing the values of $n$, we can clearly check from Table 27 that the order of Szeged and PI index is increasing while that of $\mathrm{ABC}_{\mathrm{GG}}$ and $\mathrm{NGG}$ is decreasing.

For the comparison of Szeged, PI, $\mathrm{ABC}_{\mathrm{GG}}$, and NGG index of $Q_{n}$, we computed the indices for different values of $n$. By increasing the values of $n$, we can clearly check from Table 28 that the order of Szeged and PI index is increasing while that of $\mathrm{ABC}_{\mathrm{GG}}$ and $\mathrm{NGG}$ is decreasing.

\section{Conclusion}

In this article, we have figured out several bond-additive TIs such as Szeged, PI, ABC, NGG, and Mostar index. We calculated the closed formulae for abovementioned TIs of chain silicate, oxide network, para, and ortho chain. The above outcomes contribute in the field of natural sciences 
and pharmaceutical science. Our exploration kept on determining new consequences of these graphs.

\section{Data Availability}

No data were used to support this study.

\section{Conflicts of Interest}

The authors declare that they have no conflicts of interest.

\section{References}

[1] K. Nandan, K. Ranjan, M. B. Ahmad, and B. Sah, "QSAR Studies on polychlorinated aromatic compounds using topological descriptors," International Journal of Pharmaceutical Sciences and Research, vol. 4, no. 7, p. 2691, 2013.

[2] P. J. Hansen and P. C. Jurs, "Chemical applications of graph theory. Part I. Fundamentals and topological indices," Journal of Chemical Education, vol. 65, no. 7, p. 574, 1988.

[3] I. Gutman, "A formula for the Wiener number of trees and its extension to graphs containing cycles," Graph Theory Notes New York, vol. 27, no. 9, pp. 9-15, 1994.

[4] H. Lin, "On segments, vertices of degree two and the first Zagreb index of trees," Match Communications in Mathematical and in Computer Chemistry, vol. 72, no. 3, pp. 825834, 2014.

[5] M. Črepnjak and N. Tratnik, "The szeged index and the wiener index of partial cubes with applications to chemical graphs," Applied Mathematics and Computation, vol. 309, pp. 324-333, 2017.

[6] H. Wiener, "Structural determination of paraffin boiling points," Journal of the American Chemical Society, vol. 69, no. 1, pp. 17-20, 1947.

[7] S. Nikolić and N. Trinajstić, "The Wiener index: development and applications," Croatica Chemica Acta, vol. 68, no. 1, pp. 105-129, 1995.

[8] I. Gutman and A. A. Dobrynin, "The Szeged index a success story," Graph Theory Notes New York, vol. 34, pp. 37-44, 1998.

[9] M. H. Khalifeh, H. Yousefi-Azari, and A. R. Ashrafi, "Vertex and edge PI indices of Cartesian product graphs," Discrete Applied Mathematics, vol. 156, no. 10, pp. 1780-1789, 2008.

[10] A. Graovac and M. Ghorbani, "A new version of atom-bond connectivity index," Acta Chimica Slovenica, vol. 57, no. 3, p. 609, 2010.

[11] B. Furtula, "Atom-bond connectivity index versus Graovac Ghorbani analog," Match Communications in Mathematical and in Computer Chemistry, vol. 75, pp. 233-242, 2016.

[12] D. Dimitrov, B. Ikica, and R. Škrekovski, "Remarks on the Graovac-Ghorbani index of bipartite graphs," Applied Mathematics and Computation, vol. 293, pp. 370-376, 2017.

[13] T. Došlić, I. Martinjak, R. krekovski, S. T. Spužević, and I. Zubac, "Mostar index," Journal of Mathematical Chemistry, vol. 56, no. 10, pp. 2995-3013, 2018.

[14] M. Arockiaraj, J. Clement, and N. Tratnik, "Mostar indices of carbon nanostructures and circumscribed donut benzenoid systems," International Journal of Quantum Chemistry, vol. 119, no. 1, p. 26043, 2019.

[15] F. Hayat and B. Zhou, "On cacti with large Mostar index," Filomat, vol. 33, no. 15, pp. 4865-4873, 2019.

[16] D. B. West, Introduction to Graph Theory, Prentice-Hall, Upper Saddle River, Bergen, 2001.

[17] F. Harary, Graph Theory, Addison-Wesley, Reading, MA, USA, 1969.
[18] H. Ali, M. K. Shafiq, M. R. Farahani, M. Cancan, and M. S. Aldemir, "Degree-based topological descriptors of star of david and hexagonal cage networks," Eurasian Chemical Communications, vol. 2, no. 11, pp. 1093-1100, 2020.

[19] G. Dustigeer, H. Ali, M. Imran Khan, and Y.-M. Chu, "On multiplicative degree based topological indices for planar octahedron networks," Main Group Metal Chemistry, vol. 43, no. 1, pp. 219-228, 2020.

[20] Y. Huo, H. Ali, M. A. Binyamin, S. S. Asghar, U. Babar, and J. B. Liu, "On topological indices of $\mathrm{m}$-th chain hex-derived network of third type," Frontiers of Physics, vol. 8, Article ID 593275, 2020.

[21] J. B. Liu, S. Wang, C. Wang, and S. Hayat, "Further results on computation of topological indices of certain networks," IET Control Theory \& Applications, vol. 11, no. 13, pp. 2065-2071, 2017.

[22] J.-B. Liu, M. Siddiqui, M. Zahid, M. Naeem, and A. Baig, "Topological properties of crystallographic structure of molecules," Symmetry, vol. 10, no. 7, p. 265, 2018.

[23] J.-B. Liu, J. Zhao, S. Wang, M. Javaid, and J. Cao, "On the topological properties of the certain neural networks," Journal of Artificial Intelligence and Soft Computing Research, vol. 8, no. 4, pp. 257-268, 2018.

[24] J.-B. Liu, S. Javed, M. Javaid, and K. Shabbir, "Computing first general Zagreb index of operations on graphs," IEEE Access, vol. 7, pp. 47494-47502, 2019.

[25] J.-B. Liu, M. Younas, M. Habib, M. Yousaf, and W. Nazeer, "M-Polynomials and Degree-Based Topological Indices of VC5C7 [p, q] and HC5C7 [p, q] Nanotubes," IEEE Access, vol. 7, pp. 41125-41132, 2019.

[26] P. Manuel and I. Rajasingh, "Topological properties of silicate networks," in Proceedings of the 2009 5th IEEE GCC Conference \& Exhibition, pp. 1-5, IEEE, Kuwait City, Kuwait, 2009 March.

[27] H. Ali, H. M. A. Siddiqui, and M. K. Shafiq, "On degree-based topological descriptors of oxide and silicate molecular structures," Magnet Research Report, vol. 4, pp. 135-142, 2016.

[28] A. Sadeghieh, S. Alikhani, N. Ghanbari, and A. J. M. Khalaf, "Hosoya polynomial of some cactus chains," Cogent Mathematics, vol. 4, no. 1, Article ID 1305638, 2017. 